\title{
The Expression and Significance of TIPE1 in Lichen Planus
}

\author{
Yang Peiwen, Zheng Yan*, Li Changji and Chen Caifeng \\ Department of Dermatology, the Second Affiliated Hospital of Xi'an Jiaotong University, 710004, China
}

Received: April 16, 2018; Accepted: April 25, 2018; Published: May 18, 2018

*Corresponding author: Zheng Yan, department of Dermatology, the Second Affiliated Hospital of Xi'an Jiaotong University, 157 Xiwu Road, Shaanxi Xi'an China, 10004, China, Tel: +8615319931238; E-mail: zenyan66@126.com

\begin{abstract}
Background: Lichen Planus (LP) is a common inflammatory skin disease. Studies have shown that TIPE1 may play an important role in the immune, endocrine and the formation of the tumor. However to our knowledge, there are few reports about the role of TIPE1 in inflammatory diseases.
\end{abstract}

Methods/Design: Immunohistochemistry was carried out to examine the expression of TIPE1 in tissue specimens from the lesions of 40 patients with LP, as well as from the skin of 35 normal human controls.

Results: In this study, we found that TIPE1 expression was elevated in the epidermis layer in samples of LP versus normal skin, but reduced in the liquefaction degeneration of basal layer and the lichenoid infiltration of lymphocytes in the papillary dermis.

Conclusion: Our results suggested that TIPE1 is involved in the occurrence and development of LP and that it may serve as a biomarker or therapeutic target of this disease.

Keywords: TIPE1; Lichen planus;

\section{Abbreviations}

LP: Lichen planus; TNFAIP8: Tumor necrosis factor- $\alpha$-induced protein 8; TIPE1 /TNFAIP8L1: Tumor necrosis factor- $\alpha$-induced protein 8-like 1; DEDs: Death effector domain - containing proteins; NSCLC: Non-small-cell lung cancer; HCC: Hepatocellular Carcinoma; TSC2: Tuberous sclerosis complex 2.

\section{Background}

TIPE1 (TNFAIP8L1, Tumor necrosis factor- $\alpha$-induced protein 8 -like 1) is a member of Tumor Necrosis Factor (TNF)- $\alpha$-induced protein 8 (TNFAIP8 or TIPE) family. The mammalian TNFAIP8 family consists of four members: TNFAIP8, the first identified member of this family, TNFAIP8L1 /TIPE1, TIPE2, and TIPE3, which share high degrees of sequence homology [1]. Among the members, TNFAIP8 is considered to be associated with carcinogenesis, TIPE2 is an essential negative regulator of both innate and adaptive immunity and the depletion of TIPE2 would cause serve inflammatory disease. Whereas, little is known about TIPE1 and TIPE3 [2]. TIPE1, which located in cytoplasm, was gradually begun to be studied after Cui J, et al. [3] generated a highly specific antibody to TIPE1 in 2011. Lichen Planus (LP) is a kind of idiopathic inflammatory skin disease. Its etiology is still unclear, which is considered to be related to immunity, heredity, virus infection and et al. Immunohistochemistry was carried out to examine the expression of TIPE1 in tissue specimens from the lesions of 40 patients with LP, as well as from the skin of 35 normal human controls, to explore the role of TIPE1 in the occurrence and development of lichen planus.

\section{Methods/Design}

\section{Patient Samples}

Patient samples were obtained from the tissue bank of the Department of Dermatology at the Second Affiliated Hospital of Xi'an Jiaotong University. There were a total of 75 samples collected, including 40 lichen planus (21 male and 19 female, age range $=19-66$ years), and 35 normal skin tissues (13 male and 22 female, age range $=19-56$ years) obtained from cosmetic surgery. Written informed consent for tissue procurement was obtained from all patients before study initiation, and ethics approval was obtained from the Institutional Ethics Committee of Xi'an Jiaotong University.

\section{Immunohistochemistry}

Immunohistochemical staining was performed using a standard immunoperoxidase staining procedure [4]. The staining results were evaluated under microscope by two independent pathologists and quantified based on the following scoring system. A positive rate score was first assigned according to the percentage of positive cells $(0-5 \%=0,6-25 \%=1,26-50 \%=2,51$ $75 \%=3,>75 \%=4)$. A second score of staining intensity was then assigned (colorless $=0$, light yellow $=1$, yellowish brown $=$ 2 , chocolate brown $=3$ ). The overall score for each microscopic field was calculated by the product of the two scores. The average score of five fields was taken as the final score of TIPE1 expression for each slide.

\section{Statistical Analysis}

The SPSS statistical package (SPSS, Chicago, IL, USA) was used for data analysis. The Pearson chi-square test was used for immunohistochemistry analysis, $\mathrm{P}<0.05$ was considered statistically significant. 


\section{Results}

We used immunohistochemistry to investigate TIPE1 protein expression in tissue samples from patients with lichen planus $(\mathrm{n}=40)$ and normal skin tissues $(\mathrm{n}=35)$. The results showed only cytoplasmic staining of TIPE1 (Figure1-3). For normal skin tissues, TIPE1 was weakly expressed in the basal layer and the lower stratum spinosum of the skin in $25.71 \%$ ( 9 of 35, figuer 1 ) of samples; in lichen planus, TIPE1 was mainly expressed in epidermis layer of the lesions parts, which most of the whole layer can be seen yellowish brown, and the positive rate was 62.5\% (25 of 40 , figure $\left.2, \chi^{2}=11.1189, \mathrm{P}<0.05\right)$. However, the expression

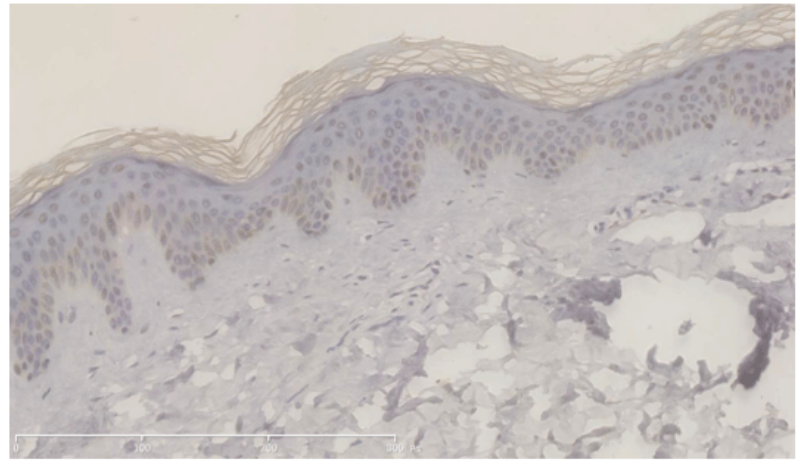

Figure 1: The expression of TIPE1 in normal skin $(\mathrm{SP} \times 200)$

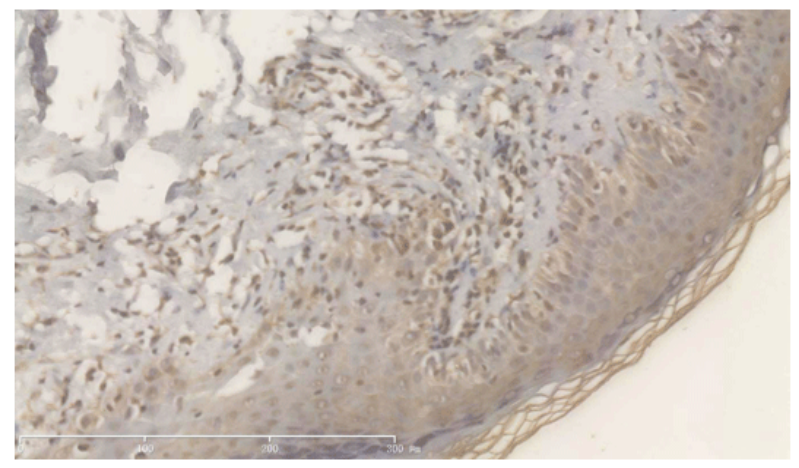

Figure 2: The positive expression of TIPE1 in skin LP $($ SP $\times 200)$

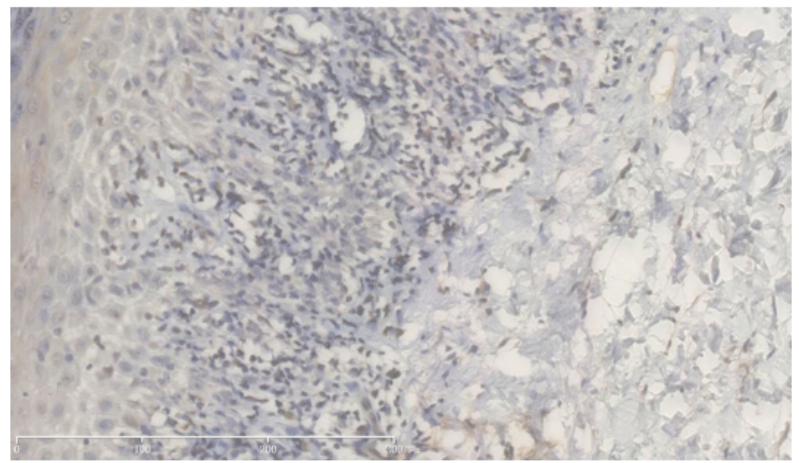

Figure 3: The negative expression of TIPE1 in skin LP $(\mathrm{SP} \times 200)$

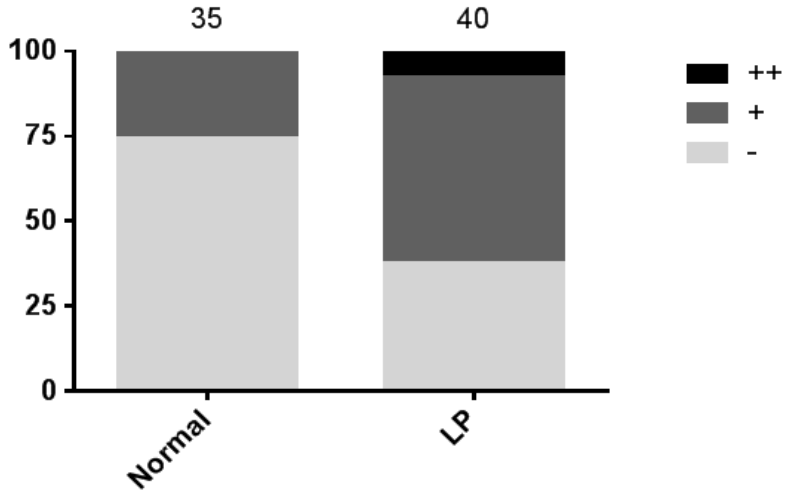

Figure 4: Semiquantitative analysis of TIPE1 staining

Table1: Expression of TIPE1 in the epidermis layer of normal skin and LP

\begin{tabular}{|c|c|c|c|c|c|c|}
\hline \multirow{2}{*}{ Group } & \multirow{2}{*}{$\mathbf{n}$} & \multicolumn{4}{|c|}{ Expression grade(n) } & Positive rate \\
\cline { 3 - 6 } & & - & + & ++ & +++ & (\%) \\
\hline LP & 40 & 15 & 22 & 3 & 0 & $62.50^{*}$ \\
\hline Normal skin & 35 & 26 & 9 & 0 & 0 & 25.71 \\
\hline Note: ${ }^{*} \mathrm{P}<0.05, \quad$ compared with normal skin \\
\hline
\end{tabular}

of TIPE1 was distinct negative in the liquefaction degeneration of basal layer and the lichenoid infiltration of lymphocytes in the papillary dermis among almost all the obtained samples of lichen planus (figure 3). In conclusion, TIPE1 expression in the epidermis layer was higher in LP than in normal skin (Table 1 and Figure 4).

\section{Discussion}

TNFAIP8 family is a newly discovered group of proteins, observed limited homologous to DEDs (Death effector domain - containing proteins).However, this novel family of proteins do not have homology with other proteins, so the TNFAIP8 family is normally considered as a new subfamily of DED containing proteins [5]. Studies have shown that the four members of TNFAIP8 family have a certain relationship with the formation of tumor and maintaining of the immune homeostasis.

TNFAIP8 / TIPE0, the first identified member, which is the most studied one of the TNFAIP8 family so far [3]. TNFAIP8 is expressed in wide variety human normal tissues, with relatively higher levels in lymphoid tissues and placenta, and lower levels in spinal cord, ovary, lung, adrenal gland, heart, brain, testis, and skeletal muscle [6]. The expression of TIPE1 is also observed among the fetal tissues in liver, lung and kidney [7]. TIPE2 was originally identified abnormally expressed in the inflamed spinal cord of mice, recently considered to be an essential negative regulator of inflammation and immune homeostasis [1,8]. TIPE1 and TIPE3 are another two uncharacterized members of TNFAIP8 family, which were identified In the last decade and considered to share a high degree of sequence homology with TIPE2 [1]. So far, little is known about TIPE1 and TIPE3. 
TIPE1, the one which we pay attention to, is located in cytoplasm, also known as $0 x i-\beta$, contains a central region with homology to death effector domains (DED-like), particularly to cFLIP DED (43\%) [2]. Unlike TIPE2, which is mainly expressed and plays the endocrine function in the immune tissues and cells, TIPE1 was expressed in wide variety tissues of mice, such as neurons in brain, hepatocytes, germ cells of reproductive organs, muscular tissues and several types of cells from the epithelial origin, particularly those with secretory functions. Also, a high level of TIPE1 mRNA was detected in most human carcinoma cell lines, especially in cells transformed with virus genomes. However, TIPE1 was not detectable in mature T or B lymphocytes, but detectable in human B lymphoblast cell line Hmy2.CIR and murine T cell line EL4 [3].

At the same time, numerous studies have shown that the TNFAIP8 family is associated with the development of multiple tumors.TNFAIP8 has been demonstrated to be upregulated in NSCLC tumor tissues, gastric adenocarcinoma tissues, platinumresistant epithelial ovarian cancer tissues, and endometrial cancer tissues [10-13]. TIPE2 expression was more pronounced in colon cancer tissues, but downregulated in HCC $[14,15]$.

As for TIPE1, Zhang, et al. [16] found that TIPE1 expression in HCC tissues showed significantly downregulated compared with adjacent non-tumor tissues, which meanwhile positively correlated with tumor pathologic grades and patient survival. The authors confirmed that TIPE1 interacted with Rac1, and inhibited the activation of Rac 1 and its downstream p 65 and c-Jun $\mathrm{N}$-terminal kinase pathway to achieve this phenomenon. These findings revealed that TIPE1 induced apoptosis in HCC cells by negatively regulating Rac1 pathway, and loss of TIPE1 might be a new prognostic indicator for HCC patients. Ha, etal. [17] discovered that TIPE1 is a novel rapamycin (mTOR) inhibitor, which is significantly up-regulated in substantia nigra dopaminergic neurons by Oxidative Stress (OS) and 6-hydroxydopamine. And up-regulated TIPE1 induces autophagy by competitive binding to FBXW5, which stabilizes Tuberous Sclerosis Complex 2 (TSC2) by preventing ubiquitination of TSC2 by CUL4-RING E3 ligase and subsequent proteasomal degradation the OS-induced TIPE1 stabilizes TSC2 protein, decreases mTOR phosphorylation, and enhances autophagy. Therefore, altered regulation of TIPE1 may contribute significantly to dysregulated autophagy observed in dopaminergic neurons under pathogenic OS condition. Wu, et al. [18] investigated the roles of TIPE1 in lung cancer, and detected lower TIPE1 mRNA and protein expression in the lung tumor tissue, compared with adjacent non-tumor tissues, which positively correlated with tumor patient survival. Liu, et al. [19] recently identified that levels of TIPE1 were significantly reduced and inversely correlated with differentiation status and distant metastasis in primary gastric cancer tissues. Mechanistically, they considered that TIPE1 medicated Wnt/ $\beta$-actin signaling which finally link TIPE1 to Epithelial-Mesenchymal Transition (EMT) inhibition. These new findings provide new evidence for the potential that TIPE1 may be an original diagnostic and therapeutic target of gastric cancer.

Existing research suggests that TIPE1, partly same as the other members of TNFAIP8 family, may play an important role in the formation of the tumor. However the role TIPE1 plays in the process how inflammatory diseases occurred and developed, still reminds to be a mystery.

Lichen planus is an inflammatory skin diseases, occurred more commonly among adults and female, favor the limbs especially flexor side, common location in trunk is waist, often accompanied with oral lesions, lesions in hair often destroy follicles and lead to a permanent hair loss. Lichen planus is a chronic skin disease, lasting months or years, the healed skin lesions may turn into residual pigmentation, depigmentation or atrophic scar. The pathologic characteristics of lichen planus is wedge-shaped hypergranulosis, irregular acanthosis with "sawtoothed" rete ridges. Liquefaction degeneration of the basal layer and lichenoid infiltrate of lymphocytes in the papillary dermis. There are multiple treatments for lichen planus, including localized widespread and oral involvement, but the most effective ones are still not recognized.

Our data showed that the expression of TIPE1 in the epidermis layer of the lesions parts in lichen planus is much higher compared with normal skin, but significantly negative in the liquefaction degeneration of basal layer and the lichenoid infiltration of lymphocytes in the papillary dermis. In conclusion, this interesting phenomenon we discovered suggests that TIPE1 may be associated with the development of lichen planus, which make it a possible therapeutic target for lichen planus. However the specific mechanism for this phenomenon remains a further research.

\section{Acknowledgments}

This work was supported by grants from the National Natural Science Foundation of China (81573055).

\section{References}

1. Sun H, Gong S, Carmody RJ, Hilliard A, Li L, Sun J, et al. TIPE2, a negative regulator of innate and adaptive immunity that maintains immune homeostasis. Cell. 2008;133(3):415-426. doi: 10.1016/j. cell.2008.03.026

2. Laliberté B, Wilson AM, Nafisi H, Mao H, Zhou YY, Daigle M, et al. TNFAIP8: a new effector for Galpha(i) coupling to reduce cell death and induce cell transformation. J Cell Physiol. 2010;225(3):865874. doi: $10.1002 /$ jcp. 22297

3. Cui J, Zhang G, Hao C, Wang Y, Lou Y, Zhang W, et al. The expression of TIPE1 in murine tissues and human cell lines. Mol Immunol. 2011;48(12-13):1548-1555. doi: 10.1016/j.molimm.2011.04.023

4. Pinheiro C, Longatto-Filho A, Scapulatempo C, Ferreira L, Martins $\mathrm{S}$, Pellerin $\mathrm{L}$, et al. Increased expression of monocarboxylate transporters 1, 2, and 4 in colorectal carcinomas. Virchows Arch. 2008;452(2):139-146. doi: 10.1007/s00428-007-0558-5

5. Tibbetts MD, Zheng L, Lenardo MJ. The death effector domain protein family: regulators of cellular homeostasis. Nat Immunol. 2003;4(5):404-409.

6. Kumar D, Gokhale P, Broustas C, Chakravarty D, Ahmad I, Kasid U. 
Expression of SCC-S2, an antiapoptotic molecule, correlates with enhanced proliferation and tumorigenicity of MDA-MB 435 cells. Oncogene. 2004;23(2):612-616.

7. Kumar D, Whiteside TL, Kasid U. Identification of a novel tumor necrosis factor-alpha-inducible gene SCC-S2, containing the consensus sequence of a death effector domain of fas-associated death domain-like interleukin-1 betaconverting enzyme-inhibitory protein. J Biol Chem. 2000;275(4):2973-2978.

8. Carmody RJ, Hilliard B, Maguschak K, Chodosh LA, Chen YH. Genomic scale profiling of autoimmune inflammation in the central nervous system: the nervous response to inflammation. J Neuroimmunol. 2002;133(1-2):95-107.

9. Shen P, Zhang H, Su Z, Wang S, Xu H. In Silico Analysis of Tumor Necrosis Factor $\alpha$-Induced Protein 8-Like-1 (TIPE1) Protein. PLoS One. 2015;10(7):e0134114. doi: 10.1371/journal.pone.0134114.

10. Wang L, Song Y, Men X. Variance of TNFAIP8 expression between tumor tissues and tumor-infiltrating CD4+ and CD8+ T cells in nonsmall cell lung cancer. Tumour Biol. 2014;35(3):2319-2325. doi: 10.1007/s13277-013-1307-9

11. Yang $M$, Zhao $Q$, Wang $X$, Liu $T$, Yao G, Lou C, et al. TNFAIP8 overexpression is associated with lymph node metastasis and poor prognosis in intestinal-type gastric adenocarcinoma. Histopathology. 2014;65(4):517-526. doi: 10.1111/his.12413

12. Liu T, Xia B, Lu Y, Xu Y, Lou G. TNFAIP8 overexpression is associated with platinum resistance in epithelial ovarian cancers with optimal cytoreduction. Hum Pathol. 2014;45(6):1251-1257. doi: 10.1016/j. humpath.2014.02.005
13. Liu T, Gao H, Yang M, Zhao T, Liu Y, Lou G. Correlation of TNFAIP8 overexpression with the proliferation, metastasis, and disease-free survival in endometrial cancer. Tumour Biol. 2014;35(6):58055814. doi: 10.1007/s13277-014-1770-y

14. Li XM, Su JR, Yan SP, Cheng ZL, Yang TT, Zhu Q. A novel inflammatory regulator TIPE2 inhibits TLR4-mediated development of colon cancer via caspase-8. Cancer Biomark. 2014;14(4):233-240.

15.Zhang YH, Yan HQ, Wang F, Wang YY, Jiang YN, Wang YN, et al. TIPE2 inhibits TNF-alpha-induced hepatocellular carcinoma cell metastasis via Erk1/2 downregulation and NF-kappaB activation. Int J Oncol. 2015;46:254-264.

16.Zhang Z, Liang X, Gao L, Ma H, Liu X, Pan Y, et al. TIPE1 induces apoptosis by negatively regulating Rac1 activation in hepatocellular carcinoma cells. Oncogene. 2015;34(20):2566-2574. doi: 10.1038/ onc.2014.208

17. Ha JY, Kim JS, Kang YH, Bok E, Kim YS, Son JH. Tnfaip8 11/Oxi-beta binds to FBXW5,increasing autophagy through activation of TSC2 in a Parkinson's disease model. J Neurochem. 2014;129(3):527-538. doi: $10.1111 /$ jnc.12643

18.Wu X, Ma Y, Cheng J, Li X, Zheng H, Jiang L, et al. TIPE1 function as a prognosis predictor and negative regulator of lung cancer. Oncotarget. 2017;8(45):78496-78506. doi: 10.18632/ oncotarget.19655

19. Liu W, Chen Y, Xie H, Guo Y, Ren D, Li Y, et al. TIPE1 suppresses invasion and migration through down-regulating Wnt/ $\beta$-catenin pathway in gastric cancer. J Cell Mol Med. 2018,22(2):1103-1117. doi: $10.1111 /$ jcmm.13362 